\title{
Dissipative hydrodynamics for multi-component systems
}

\author{
Andrej $\mathrm{El}^{1, \mathrm{a}}$, Ioannis Bouras ${ }^{1}$, Christian Wesp ${ }^{1}$, Zhe $\mathrm{Xu}^{2}$, and Carsten Greiner ${ }^{1}$ \\ 1 Institut für Theoretische Physik, Goethe Universität, Max-von-Laue Str. 1, 60438 Frankfurt am Main, Germany \\ 2 Department of Physics, Tsinghua University, Beijing 100084, China
}

Received: 12 June 2012 / Revised: 3 October 2012

Published online: 27 November 2012

(c) The Author(s) 2012. This article is published with open access at Springerlink.com

Communicated by T. Bíró

\begin{abstract}
Second-order dissipative hydrodynamic equations for each component of a multi-component system are derived using the entropy principle. Comparison of the solutions with kinetic transport results demonstrates validity of the obtained equations. We demonstrate how the shear viscosity of the total system can be calculated in terms of the involved cross-sections and partial densities. The presence of the interspecies interactions leads to a characteristic time dependence of the shear viscosity of the mixture, which also means that the shear viscosity of a mixture cannot be calculated using the Green-Kubo formalism the way it has been done recently. This finding is of interest for understanding of the shear viscosity of a quark-gluon plasma extracted from comparisons of hydrodynamic simulations with experimental results from RHIC and LHC.
\end{abstract}

\section{Introduction}

The deconfined state of QCD matter produced at the early stage of ultrarelativistic heavy-ion collisions at RHIC and LHC is a multi-component system with quark and gluon degrees of freedom. Large values of the elliptic flow coefficient $v_{2}$, observed at RHIC $[1,2]$ and LHC [3], indicate that the produced quark-gluon plasma (QGP) is a nearly perfect fluid. This has motivated rapid developments on relativistic dissipative hydrodynamic formalisms [4-10]. The value of the shear viscosity to the entropy density ratio $\eta / s$ for the QGP at RHIC and LHC was extracted from comparisons of hydrodynamic [11-15] as well as kinetic transport [16] calculations with experimental data. All these hydrodynamic formalisms are based on the assumption that the quark-gluon mixture can be regarded as an effective one-component system, where $\eta / s$ is an external parameter characterizing the dissipation in the system. For a one-component system the $\eta / s$ ratio can be calculated using the Green-Kubo formalism [17-19] as well as other systematic approaches [20,21]. One may ask the question whether analogous calculation of the shear viscosity of a mixture is possible, i.e. whether a mixture behavior is equivalent to a one-component system or rather not. These questions are of major interest for investigation of the QGP properties.

In this paper we demonstrate that a standard onecomponent hydrodynamic description with a single shear

\footnotetext{
${ }^{a}$ e-mail: el@th.physik.uni-frankfurt.de
}

viscosity coefficient calculated by, e.g., Green-Kubo formalism in general cannot be applied to a multi-component system. We will explain this statement by deriving secondorder dissipative hydrodynamic equations for a multicomponent system from the entropy principle. Our approach differs from the one reported in ref. [10], since we introduce separate evolution equations and transport coefficients for each component of the mixture. We then show that by summing up equations for all components one can obtain an equation for the system as a whole, which has a relaxation-type form characteristic for all the present hydrodynamic formalisms, but the effective shear viscosity for the mixture is now related to the partial shear pressures of its components and thus has a non-trivial time dependence which is not supported by the Green-Kubo or, for this matter, any other formalism, in which an equilibrium state of matter is assumed. We will confirm our findings by calculating the shear viscosity of a mixture using the Green-Kubo formula and comparing solutions of the hydrodynamic equations with the ones from kinetic transport calculations.

\section{Dissipative hydrodynamic formalism for a multi-component system}

We consider a mixture of $N$ particle species, for which we define a common velocity field $u^{\mu}$. Neglecting bulk pressure and heat flow we can construct the total entropy cur- 
rent as [22]

$$
s^{\mu}=\sum_{i=1}^{N} s_{i}^{\mu}=s_{e q} u^{\mu}-\sum_{i=1}^{N} \frac{\beta_{i}}{2 T_{i}} \pi_{i, \alpha \beta} \pi_{i}^{\alpha \beta} u^{\mu},
$$

where $s_{e q}$ is the total entropy density in local equilibrium and $u^{\mu}$ is the hydrodynamic velocity. $T_{i}$ and $e_{i}$ are the temperature and local energy density of the particle species $i$. In analogy to the one-component case $[7,21]$ one obtains $\beta_{i}=\left(9 / 4 e_{i}\right) . \pi_{i}^{\mu \nu}=T_{i}^{\mu \nu}-T_{i, e q}^{\mu \nu}$ is the shear stress tensor, which is, as long as heat flow and bulk pressure are neglected, the difference between the energy-momentum tensor $T_{i}^{\mu \nu}$ and the equilibrium one. Equation (1) is the generalization of the entropy current for a one-component system $(N=1)$, discussed, for instance, in refs. [4-7,21]. By introducing different temperatures $T_{i}$ for each species in eq. (1) we consider the most general situation in which the mixture components did not achieve a common (pre-) equilibrium state. The temperatures $T_{i}$ as well as the energy densities $e_{i}$ must be considered dynamical quantities.

The total entropy production is then

$$
\begin{aligned}
\partial_{\mu} s^{\mu}= & \sum_{i} \pi_{i, \alpha \beta} \\
& \times\left[\frac{\sigma^{\alpha \beta}}{T_{i}}-\pi_{i}^{\alpha \beta} \partial_{\mu}\left(\frac{\beta_{i}}{2 T_{i}} u^{\mu}\right)-\frac{\beta_{i}}{T_{i}} u^{\mu} \partial_{\mu} \pi_{i}^{\alpha \beta}\right],
\end{aligned}
$$

with the shear tensor

$$
\begin{aligned}
\sigma^{\mu \nu} & =\nabla^{\langle\mu} u^{\nu\rangle} \\
& =\left(\frac{1}{2}\left(\Delta_{\alpha}^{\mu} \Delta_{\beta}^{\nu}+\Delta_{\alpha}^{\nu} \Delta_{\beta}^{\mu}\right)-\frac{1}{3} \Delta_{\alpha \beta} \Delta^{\mu \nu}\right) \nabla^{\alpha} u^{\alpha}
\end{aligned}
$$

and $\Delta_{\alpha \beta}=g_{\alpha \beta}-u_{\alpha} u_{\beta}$ with the metric $g_{\alpha \beta}=$ $\operatorname{diag}(1,-1,-1,-1)$. We have used conservation of the partial particle flows and total energy-momentum tensor, $\partial_{\mu} N_{i}^{\mu}=0$ and $\partial_{\mu} T^{\mu \nu}=0$, to obtain eq. (2). The obtained equation for the entropy balance is again a generalization of the one-component result [7].

According to the second law of thermodynamics the entropy production is non-negative. A simple way to fulfill this is to make the terms in the square bracket in eq. (2) to be proportional to $\pi_{i}^{\alpha \beta},[\ldots]=\pi_{i}^{\alpha \beta} /\left(2 \eta_{i} T_{i}\right)$. Then the entropy production (2) has the following algebraic structure:

$$
\partial_{\mu} s^{\mu} \stackrel{!}{=} \sum_{i=1}^{N} \frac{\pi_{i, \alpha \beta} \pi_{i}^{\alpha \beta}}{2 \eta_{i} T_{i}} \geq 0 .
$$

This leads to the dynamical equation for each $\pi_{i}^{\alpha \beta}$,

$$
u^{\mu} \partial_{\mu} \pi_{i}^{\alpha \beta}=-\frac{\pi_{i}^{\alpha \beta}}{2 \eta_{i} \beta_{i}}-\pi_{i}^{\alpha \beta} \frac{T_{i}}{\beta_{i}} \partial_{\mu}\left(\frac{\beta_{i}}{2 T_{i}} u^{\mu}\right)+\frac{\sigma^{\alpha \beta}}{\beta_{i}},
$$

which is analogous to the equation introduced by Israel and Stewart for a one-component system $(N=1)[4-7]$.

In order to apply eq. (4) to a multi-component system $(N>1)$, we first need to determine the coefficients $\eta_{i}$, which in general differ from the usual definition of the shear viscosity. The reason for this is that $\pi_{i}^{\alpha \beta}$,s are correlated due to interactions between particles from different species. These correlations between $\pi_{i}^{\alpha \beta}$ 's can only be seen, when each $\eta_{i}$ depends on all $\pi_{j}^{\alpha \beta}, j=1,2, \ldots, N$. We will also show later that the coefficients $\eta_{i}$ become the shear viscosities, when the ratios of components of $\pi_{i}^{\alpha \beta}$, $\mathrm{s}$ are relaxing to constants in time.

We now make use of relativistic kinetic theory and express the entropy current via the phase-space distribution function $f_{i}\left(x, p_{i}\right)$,

$$
s^{\mu}=\sum_{i=1}^{N} \int \mathrm{d} \Gamma_{i} p_{i}^{\mu} f_{i}\left(x, p_{i}\right)\left[1-\ln f_{i}\left(x, p_{i}\right)\right],
$$

with $\mathrm{d} \Gamma_{i}=\mathrm{d}^{3} p_{i} / E_{i} /(2 \pi)^{3}$. It was shown for the case of $N=1[23]$ and is obviously true for $N>1$ that using the Grad's ansatz [7],

$$
f_{i}\left(x, p_{i}\right)=f_{i, e q}\left(x, p_{i}\right)\left(1+A_{i} \pi_{i, \mu \nu} p_{i}^{\mu} p_{i}^{\nu}\right),
$$

in eq. (5) one obtains eq. (1) up to second order in $\pi_{i, \mu \nu}$. Here $f_{i, e q}\left(x, p_{i}\right)$ is the equilibrium distribution function and $A_{i}=\left[2\left(e_{i}+P_{i}\right) T_{i}^{2}\right]^{-1}[21]$, where $P_{i}$ is the pressure.

The space-time evolution of $f_{i}\left(x, p_{i}\right)$ obeys the Boltzmann equation

$$
p_{i}^{\mu} \partial_{\mu} f_{i}=C_{i}\left[f_{1}, f_{2}, \ldots, f_{N}\right]=C_{i i}\left[f_{i}\right]+\sum_{j=1, j \neq i}^{N} C_{i j}\left[f_{i}, f_{j}\right],
$$

where $C_{i i}$ are the collision terms describing interactions of particles of same species and $C_{i j}$ describing binary interactions of particles of different species. Explicit expressions for the collision terms can be found, for example, in $[24,25]$. Taking derivative of (5) and using (7) we obtain

$$
\partial_{\mu} s^{\mu}=\sum_{i=1}^{N} A_{i} \pi_{i, \mu \nu} \int \mathrm{d} \Gamma_{i} p_{i}^{\mu} p_{i}^{\nu} C_{i} .
$$

Comparison between eqs. (8) and (3) leads to

$$
\eta_{i}=\frac{\pi_{i, \mu \nu} \pi_{i}^{\mu \nu}}{2 A_{i} \pi_{i, \mu \nu} \int \mathrm{d} \Gamma_{i} p_{i}^{\mu} p_{i}^{\nu} C_{i}} .
$$

Because the collision term $C_{i}$ is a functional of all $f_{j}$ 's, each $\eta_{i}$ depends on all $\pi_{j}^{\mu \nu}$ 's with $j=1,2, \ldots, N$.

In order to simplify eq. (9) we will now consider a onedimensional system, which implies that the shear stress tensor has a diagonal (and of course traceless) form: $\pi^{\alpha \beta}=$ $\operatorname{diag}(0, \pi / 2, \pi / 2,-\pi)$, with the single independent component $\pi$. By virtue of eq. (6) this form of the shear stress tensor is equivalent to a deformation of the momentumspace distribution along the $z$-axis, whereas in the transverse $x y$ plain the momentum distribution is isotropic. Furthermore we consider only two species, $i, j=1,2$ and isotropic scattering processes, i.e. we assume that the differential cross-section $\mathrm{d} \sigma / \mathrm{d} \Omega$ does not depend on the scattering angle. Inserting the off-equilibrium destribution (6) 
into the collision term in (9) and using the aforementioned simplifications we obtain the following expression for the shear viscosities of the mixture constituents:

$$
\eta_{i}^{-1}=T_{i}^{-1} \sum_{j=1}^{N}\left(\frac{7}{6} \frac{n_{j}}{n_{i}}-\frac{1}{3} \frac{\pi_{j}}{\pi_{i}}\right) \sigma_{i j} .
$$

In the latter equation $n_{j} / n_{i}$ denotes the ratio of the particle densities of mixture constituents. In our formalism this chemical composition is fixed by the initial consition and does not change in time, although this assumption is a very strong simplification for a QGP. Now the obtained expression for $\eta_{i}$ can be inserted into the dynamic evolution equation (4) for $\pi_{i}$ (that is we take the $\pi_{33}$ component of $\pi_{\mu \nu}$ in (4), although considering any other component will lead to identical result). We obtain a dynamic equation for the shear tensor components $\pi_{i}$ in a mixture,

$$
\begin{aligned}
\dot{\pi}_{i}= & -\left(\frac{5}{9} n_{i} \sigma_{i i}+\frac{7}{9} n_{j} \sigma_{i j}\right) \pi_{i}+\frac{2}{9} n_{i} \sigma_{i j} \pi_{j} \\
& -\pi_{i} \frac{T_{i}}{\beta_{i}} \partial_{\mu}\left(\frac{\beta_{i}}{2 T_{i}} u^{\mu}\right)+\frac{\sigma}{\beta_{i}},
\end{aligned}
$$

with the short-hand notation $\dot{\pi} \equiv u_{\mu} \partial^{\mu} \pi$ and $\sigma$ denoting the $z z$ component of the shear tensor $\sigma=\sigma^{33}$. A distinct feature of the obtained equation, which has a characteristic relaxation time form, is the presence of two time scales for relaxation, of which the second one is associated with a coupling between the partial shear pressures $\pi_{i}$ of the two species.

\section{Coupled dynamics in a mixture}

Now the question arises, as of how the dependence of $\eta_{i}$ in eq. (10) on the ratio $\pi_{i} / \pi_{j}$ can be interpreted. If $\eta_{i}$ is understood as the shear viscosity of the medium, it must be a property of the medium, i.e. depend exclusively on its chemical composition and the associated cross-sections. Moreover, the shear viscosity must be defined in the proximity of the equilibrium. Let us consider the following situation: all velocity gradients vanish. This means that the shear tensor $\sigma^{\mu \nu}$, and thus $\sigma$ as well, vanish. We also assume the the temperatures of the two sub-systems are equal and can be replaced by the single temperature $T$ of the system, i.e. $T_{i}=T$. This assumption is necessary since we will not consider balance equations for the partial energy densities $e_{i}$. These equations must be obtained from the partial energy-momentum tensor divergence $\partial_{\mu} T_{i}^{\mu \nu}=J_{i}^{\nu}$ with the source terms $J_{i}^{\nu}$ describing energy-momentum exchange between the species. Since we will not attempt to derive the source terms $J_{i}^{\nu}$ in scope of this work, only the conservation equation for the total energy-momentum tensot $\partial_{\mu} T^{\mu \nu}=0$ can be used, from which evolution equations for the total energy density $e$ and for the total effective temperature $T=e /(3 n)$ are obtained. By assuming $T_{i}=T$ we thus overcome the need to specify balance equations for the partial energy densities and temperatures.
We move into the fluid rest frame, in which $u_{\mu} \partial^{\mu} \equiv$ $\partial / \partial \tau$. Equations (11) are now reduced to a set of two coupled differential equations for the shear stress tensor components $\pi_{1}, \pi_{2}$ with the parameters $n_{1}, n_{2}, \sigma_{11}, \sigma_{12}$ and $\sigma_{22}$. The system of equations can be solved analytically and thus a solution for $\frac{\pi_{1}}{\pi_{2}}(\tau)$ is also found,

$$
\frac{\pi_{1}}{\pi_{2}}(\tau)=\mathcal{A}(n, \sigma) \cdot \tanh \left(\tau \cdot \mathcal{B}(n, \sigma)+\mathcal{D}\left(n, \sigma, \pi_{0}\right)\right) .
$$

In the latter equation $\mathcal{A}, \mathcal{B}$ and $\mathcal{D}$ are algebraic functions of the system properties $n_{i}$ and $\sigma_{i j}$, and in particular $\mathcal{D}$ depends on the initial value of the ratio $\pi_{1} / \pi_{2}$. Equation (12) demonstrates that the ratio $\pi_{1} / \pi_{2}$ of the partial shear pressures is determined completely by the properties of the system, but is not a constant. Moreover, eq. (12) leads to saturation of $\pi_{1} / \pi_{2}$ in $\tau \rightarrow \infty$ limit, i.e. a welldefined characteristic limit for the ratio exists. In general this characteristic value is different from the value $n_{1} / n_{2}$. It is also important to mention, that there is no conclusive way to specify the initial values of $\pi_{1}$ and $\pi_{2}$ - or just $\pi$ for a standard one-component hydrodynamic calculation. In most hydrodynamic approaches the standard choice is therefore the trivial initialization $\pi\left(\tau_{0}\right)=0$. Another possible choice is initialization with the Navier-Stokes value $\pi\left(\tau_{0}\right)=2 \eta \sigma[15]$. As it is for the one-component case, for a multi-component system the choice of the initial condition for $\pi_{i}$ is not clear as well. If the gradients are "switched on", the trivial choice $\pi_{i}\left(\tau_{0}\right)=0$ will lead, according to eq. (11) to $\dot{\pi}_{i}=\sigma / \beta_{i}$. And since $\beta_{2} / \beta_{1}=e_{1} / e_{2}=n_{1} / n_{2}$, after a short time the shear pressure ratio will be approximately equal to the density ratio, $\pi_{1} / \pi_{2} \approx n_{1} / n_{2}$.

It is also interesting to build the sum of eqs. (11), which leads us to a relaxation equation for the total shear pressure in the mixture $\dot{\pi}=\dot{\pi}_{1}+\dot{\pi}_{2}$. If we write this equation in the relaxation time form with only one relaxation scale, as is usual for most hydrodynamic formalisms presently used [11-15], we obtain

$$
\begin{aligned}
\dot{\pi}= & -\pi \cdot \frac{5}{9}\left(\frac{\pi_{1} / \pi_{2}}{1+\pi_{1} / \pi_{2}} \cdot\left(n_{1} \sigma_{11}+n_{2} \sigma_{12}\right)\right. \\
& \left.+\frac{1}{1+\pi_{1} / \pi_{2}} \cdot\left(n_{2} \sigma_{22}+n_{1} \sigma_{12}\right)\right)+ \text { gradients. }
\end{aligned}
$$

According to the standard definition of the relaxation time $\tau_{\pi}$ in the second-oder formalisms [4-7],

$$
\tau_{\pi}=\frac{9 \eta}{2 e}
$$

we recognize that the shear viscosity of the mixture is now given by

$$
\eta_{\text {mix }}=\frac{2}{5} e\left(\frac{\pi_{1} / \pi_{2}}{1+\pi_{1} / \pi_{2}} \cdot \lambda_{1}^{-1}+\frac{1}{1+\pi_{1} / \pi_{2}} \cdot \lambda_{2}^{-1}\right)^{-1}
$$

with the inverse of the mean free path $\lambda_{1}^{-1}=n_{1} \sigma_{11}+n_{2} \sigma_{12}$ and $\lambda_{2}^{-1}=n_{2} \sigma_{22}+n_{1} \sigma_{12}$ and the time-dependent ratio $\pi_{1} / \pi_{2}$ given by eq. (12). 
The obtained result for the shear viscosity of the mixture is interesting for the following reason. If one attempts to calculate the shear viscosity of the mixture we have considered here, e.g., using the Green-Kubo formula in a kinetic transport simulation, the result would naturally be a constant value for $\eta_{m i x}$. On the other hand the result obtained here in form of eq. (15) implies that the viscosity of a mixture $i s$ time dependent, but saturates. The value at which the time dependence dies off will not be identical with the value one would obtain using the Green-Kubo formalism because the coupling between the species in a mixture induces an internal dynamics in a system.

To demonstrate this, we calculate the shear viscosity coefficient of a mixture in the kinetic transport model Boltzmann Approach to MultiParton Scatterings (BAMPS) $[24,25,16]$ using the procedure successfully applied by us in ref. [17]. The cross-sections for the scattering processes of the two species, confined in a static box, are chosen to be $\sigma_{11}=10 \mathrm{GeV}^{-2}, \sigma_{12}=5 \mathrm{GeV}^{-2}$ and $\sigma_{22}=2.5 \mathrm{GeV}^{-2}$. The density ratio is $n_{1} / n_{2}=5$. The temperature is chosen to be $T=0.4 \mathrm{GeV}$ and both particle species are considered to be Boltzmann gases with degeneracy factors 16 , i.e. $n_{1}=5 / 6 \cdot 16 / \pi^{2} T^{3}$ and $n_{2}=1 / 6 \cdot 16 / \pi^{2} T^{3}$. The equation of state is the ideal one, i.e. $e_{1}=3 n_{1} T$ and $e_{2}=3 n_{2} T$. Note that for this setup the mean-free path scales for the two species are $\lambda_{1}=0.207 \mathrm{fm}$ and $\lambda_{2}=0.414 \mathrm{fm}$. These values are chosen to crudely simulate quarks and gluons in a QGP. To obtain the shear viscosity, we extract the correlation function,

$$
\begin{aligned}
C(\tau)= & \frac{1}{3}\left(\left\langle\pi^{x y}(0) \pi^{x y}(\tau)\right\rangle\right. \\
& \left.+\left\langle\pi^{x z}(0) \pi^{x z}(\tau)\right\rangle+\left\langle\pi^{y z}(0) \pi^{y z}(\tau)\right\rangle\right),
\end{aligned}
$$

where $\tau$ is the correlation time and $\langle$.$\rangle denotes ensemble$ average in the static box. The Green-Kubo formalism relates the shear viscosity to the integral of the correlation function over the relaxation time,

$$
\eta=\frac{V}{T} \int_{0}^{\infty} C(\tau) \mathrm{d} \tau,
$$

where $V$ denotes the considered volume and $T$ the temperature of the system. In a standard one-component case the correlator $C(\tau)$ is very good described by an exponential function with the relaxation time $\tau_{c}$ [17-19]. For a mixture, however, we find that a single-exponent fit does not work and two-exponent fit must be considered,

$$
C(\tau)=C_{1} \cdot e^{-\tau / \tau_{1}}+C_{2} \cdot e^{-\tau / \tau_{2}},
$$

with two relaxation times $\tau_{1}$ and $\tau_{2}$. In fig. 1 we demontrate the correlation function extracted from BAMPS static box calculations together with the two-exponetial fit. Integrating the correlation function shown in fig. 1 over the correlation time we obtain for the shear viscosity $\eta=0.062 \mathrm{GeV}^{-3}$. To demonstrate the difference between this result and the effective mixture viscosity (15) we show in fig. 2 the time evolution of $\eta_{m i x}$ for the setup described

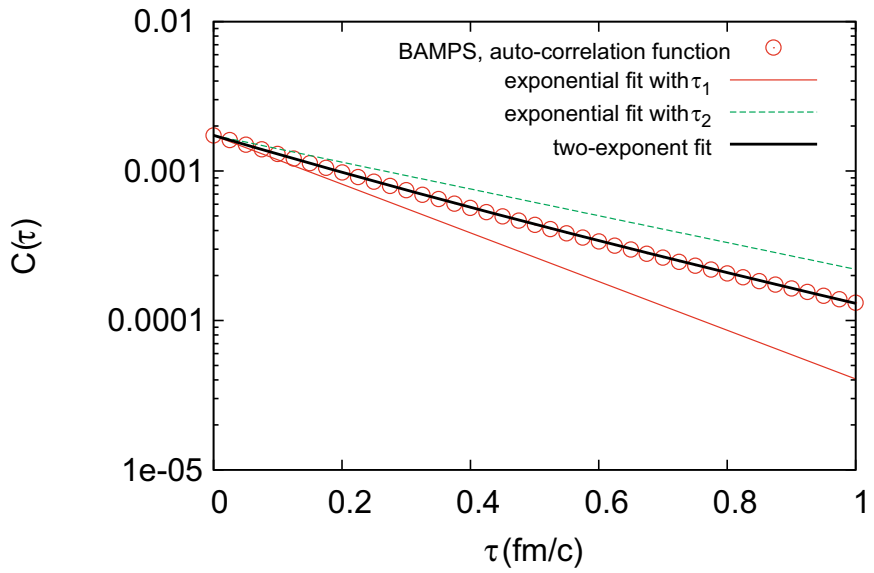

Fig. 1. Autocorrelation function extracted from BAMPS (symbols) as function of correlation time. The two-exponent fit eq. (18) with $C_{1}=C_{2}=8.66 \cdot 10^{-4} \mathrm{GeV}^{2} / \mathrm{fm}^{6}, \tau_{1}=$ $0.264822 \mathrm{fm} / c$ and $\tau_{2}=0.479556 \mathrm{fm} / c$ is shown by bold solid line. The two exponents from the two-exponent fit are also shown separately by the thin solid and dashed lines.

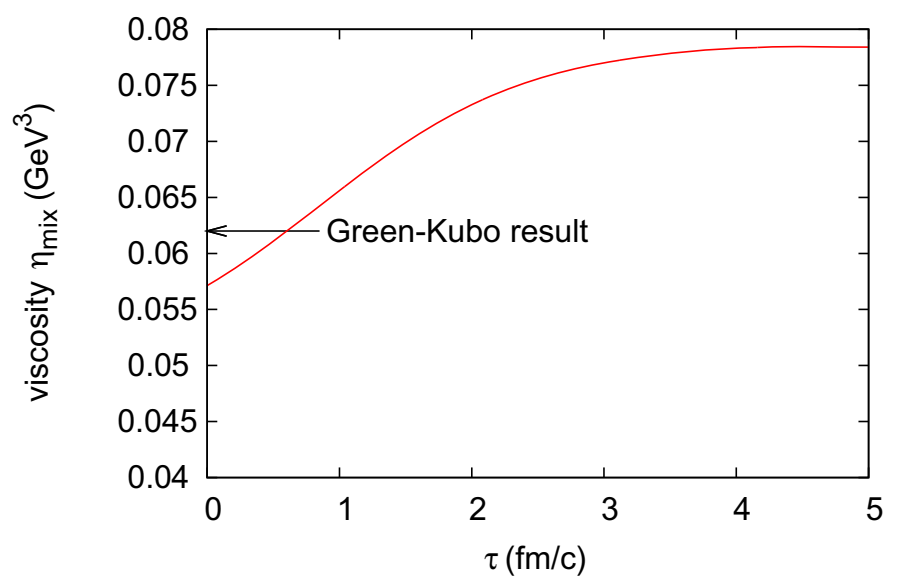

Fig. 2. Time evolution of the shear viscosity $\eta_{m i x}$ from eq. (15). See the text for details of the setup. The result of the Green-Kubo formalism is indicated by the arrow.

above. From fig. 2 we recognize that the mixture viscosity $\eta_{m i x}$ is approximately equal to the Green-Kubo result at early evolution stage but significantly increases with time. In order to verify the applicability of the obtained relaxation-type equations (11) for the shear pressure we now study relaxation of shear pressure in a static BAMPS box. We use a quasi-static setup, i.e. a volume with no gradients but finite initial shear pressures $\pi_{1}(0)$ and $\pi_{2}(0)$. In the kinetic transport solver BAMPS this is achieved by sampling particles isotropically in space according to the distribution function (6) with a chosen value of $\pi=\pi^{z z}$. This setup provides a cross-check of the relaxation dynamics described by the first two terms on the right-hand side of eqs. (11) and also provides a possibility to cross-check of the validity of eq. (12). We use same cross-sections and composition of the gas as for the Green-Kubo calculations 


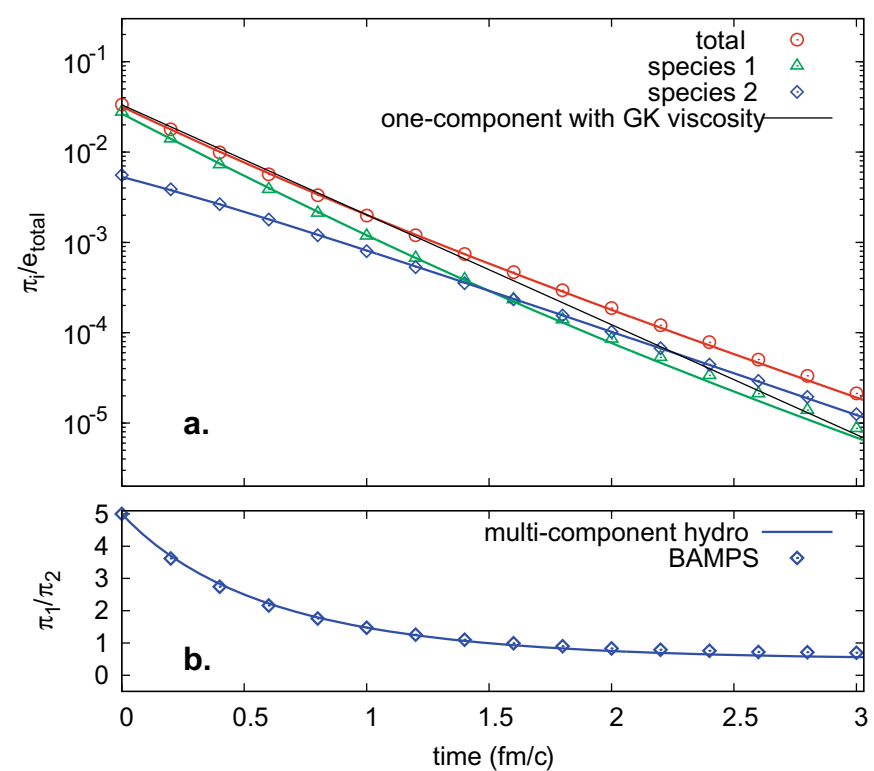

Fig. 3. Relaxation of the total and partial shear pressures in BAMPS (symbols) and analytic solutions (lines). Additionally, the one-component solution with the viscosity obtained using the Green-Kubo formalism is shown to reproduce the total shear pressure of a multi-component system only at early times of the evolution.

discussed above. Results of BAMPS static box calculations are shown in fig. 3. The BAMPS results (symbols) for the total as well as the partial shear pressures (fig. 3 (a)) are well reproduced by the analytic solutions of eq. (11). The ratio $\pi_{1} / \pi_{2}$ calculated in BAMPS is also shown to agree with the analytic solution and demonstrates the expected saturation (fig. 3 (b)). The one-component solution $\pi(\tau)=\pi\left(\tau_{0}\right) \cdot e^{-\tau / \tau_{\pi}}$ with the shear viscosity value $\eta_{G K}$ calculated using Green-Kubo formalism is shown by the solid black line in fig. 3 (a) and is unable to describe relaxation of the total shear pressure in a multi-component system on a long time scale. This means that the GreenKubo formalism in the form it is applied to one-component systems cannot be applied to calculate the shear viscosity of a mixture. From fig. 3 (a) we rather recognize that the viscosity must increase with time, though it might be close to the Green-Kubo result at early times of the evolution. The observed failure of the standrad Green-Kubo formalism implies that an appropriate formulation of the Green-Kubo relation for mixtures is needed. This is as well emphasized by fig. 1 and eq. (18), which demonstrate that the behaviour of the auto-correlation function is different in a one- and multi-component systems.

\section{Conclusions}

We have derived second-order hydrodynamic equations for the shear tensor components of constituents of a mixture. A cross-check of the obtained equations is provided by comparisons of the solutions with kinetic transport calculations with BAMPS, which demonstrate very good agree- ment of the results. We have also demonstrated that the effective shear viscosity of a mixture of two components does have a non-trivial time dependence, which is explained by inner dynamics of the mixture due to inter-species interactions. If the Green-Kubo formalism is applied to calculate the shear viscosity of a mixture, the result cannot capture its time dependence. Thus, if calculated by the GreenKubo formalism, the shear viscsosity cannot be used to describe hydrodynamic evolution of a mixture. It will be very interesting to investigate the impact of our finding on extraction of the shear viscosity of a quark-gluon mixture from comparisons of experimental data with the results of dissipative hydrodynamic simulations. We expect for example, that the elliptic flow coefficient $v_{2}\left(p_{T}\right)$ calculated from a mixture, such as the quark-gluon plasma, cannot be accurately reproduced by a one-component dissipative hydrodynamic calculations unless the proper time dependence of the shear viscosity, which we have introduced in this paper, is taken into account.

This work was supported by the Helmholtz International Center for FAIR within the framework of the LOEWE program launched by the State of Hesse. IB and CW acknowledge support by HGS-Hire. The authors are greatful to the Center for Scientific Computing (CSC) at Frankfurt University and LOEWE-CSC for the computing resources.

Open Access This is an open access article distributed under the terms of the Creative Commons Attribution License (http://creativecommons.org/licenses/by/3.0), which permits unrestricted use, distribution, and reproduction in any medium, provided the original work is properly cited.

\section{References}

1. PHENIX Collaboration (S.S. Adler et al.), Phys. Rev. Lett. 91, 182301 (2003) nucl-ex/0305013.

2. S.A. Voloshin, A.M. Poskanzer, R.Snellings, arXiv:0809.2949 [nucl-ex].

3. The ALICE Collaboration (K. Aamodt et al.), arXiv:1011.3914 [nucl-ex].

4. W. Israel, Ann. Phys. (N.Y.) 100, 310 (1976).

5. J.M. Stewart, Proc. R. Soc. London, Ser. A 357, 59 (1977).

6. W. Israel, M. Stewart, Ann. Phys. (N.Y.) 118, 341 (1979).

7. A. Muronga, Phys. Rev. C 69, 034903 (2004). arXiv:0309055 [nucl-th].

8. P. Romatschke, Int. J. Mod. Phys. E 19, 1 (2010) arXiv:0902.3663 [hep-ph].

9. G.S. Denicol, T. Koide, D.H. Rischke, Phys. Rev. Lett. 105, 162501 (2010) arXiv:1004.5013 [nucl-th].

10. A. Monnai, T. Hirano, Nucl. Phys. A 847, 283 (2010) arXiv:1003.3087 [nucl-th].

11. H. Song, U.W. Heinz, Phys. Rev. C 77, 064901 (2008) arXiv:0712.3715 [nucl-th].

12. M. Luzum, P.Romatschke, Phys. Rev. C 78, 034915 (2008) 79, 039903(E) (2009) arXiv:0804.4015 [nucl-th].

13. D.A. Teaney, arXiv:0905.2433 [nucl-th].

14. H. Song, S.A. Bass, U.W. Heinz arXiv:1103.2380 [nucl-th].

15. H. Niemi, G.S. Denicol, P. Huovinen, E. Molnar, D.H. Rischke, arXiv:1203.2452 [nucl-th]. 
16. Z. Xu, C. Greiner, H. Stöcker, Phys. Rev. Lett. 101, 082302 (2008) arXiv:0711.0961 [nucl-th].

17. C. Wesp, A. El, F. Reining, Z. Xu, I. Bouras, C. Greiner, Phys. Rev. C 84, 054911 (2011) arXiv:1106.4306 [hep-ph].

18. N. Demir, S.A. Bass, Phys. Rev. Lett. 102, 172302 (2009) arXiv:0812.2422 [nucl-th].

19. J.I. Fuini, N.S. Demir, D.K. Srivastava, S.A. Bass, J. Phys. G 38, 015004 (2011) arXiv:1008.2306 [nucl-th].

20. F. Reining, I. Bouras, A. El, C. Wesp, Z. Xu, C. Greiner, Phys. Rev. E 85, 026302 (2012) arXiv:1106.4210 [hep-th].
21. A. El, A. Muronga, Z. Xu, C. Greiner, Phys. Rev. C 79, 044914 (2009) arXiv:0812.2762 [hep-ph].

22. M. Kranyš, Arch. Ration. Mech. Anal. 39, 245 (1970).

23. A. El, Z. Xu, C. Greiner, Phys. Rev. C 81, 041901 (2010) arXiv:0907.4500 [hep-ph].

24. Z. Xu, C. Greiner, Phys. Rev. C 71, 064901 (2005) arXiv:0406278 [hep-ph].

25. Z. Xu, C. Greiner, Phys. Rev. C 76, 024911 (2007) arXiv:hep-ph/0703233. 